Check for updates

Cite this: RSC Adv., 2018, 8, 15158

Received 20th October 2017

Accepted 3rd April 2018

DOI: 10.1039/c7ra11610a

rsc.li/rsc-advances

\section{The enhanced angiogenesis effect of VEGF-silk fibroin nanospheres-BAMG scaffold composited with adipose derived stem cells in a rabbit model}

\author{
Dongliang Zhang, (D) Nailong Cao, Shukui Zhou, Zhong Chen, Xinru Zhang \\ and Weidong Zhu (iD *
}

We report a study to determine whether a vascular endothelial growth factor (VEGF)-silk fibroin (SF) nanospheres-bladder acellular matrix graft (BAMG) scaffold composited with adipose derived stem cells (ADSCs) could enhance angiogenesis in bladder regeneration in rabbits. Rabbit ADSCs were isolated and identified by flow cytometry. The morphology and release behaviour of VEGF-SF nanospheres were detected. After the composite scaffolds were successfully used in bladder reconstruction, the bladder capacity, H\&E staining and immunohistochemical staining were studied at different time points. ADSCs exerts high expression rates of CD29, CD90, and CD44, accompanied with low expression rates of CD34 and CD45. SF nanospheres with diameters of 200-1000 nm were prepared to load VEGF, and they contributed to maintain the release of VEGF. The reconstructed bladder with VEGF-SF nanospheresBAMG plus ADSCs had more regular smooth muscle tissue and blood vessels. Moreover, instead of differentiating into epithelial or vascular endothelial cells, ADSCs may be more likely to provide additional cytokines to enhance angiogenesis in the bladder regeneration process. The tissue engineered bladder constructed by BAMG modified by VEGF-SF nanospheres possessed high bio-compatibility and an enhanced angiogenesis effect, and could be used as an ideal biological material to repair bladder defects after being composited with ADSCs.

\section{Introduction}

Conventional bladder reconstruction with gastrointestinal tissues always brings new trauma to the body, and is accompanied by a series of severe complications, such as electrolyte disturbance, urolithiasis, and vesicoureteral reflux. ${ }^{1}$ Tissue engineering technology may avoid most of these complications and provide a new therapeutic approach to bladder reconstruction. ${ }^{2}$ Since the successful application of tissue-engineered autologous bladders in patients with neurogenic bladder dysfunction, ${ }^{3}$ increasing numbers of researchers have sought to achieve their goals using various artificial or natural tissues. ${ }^{4,5}$ Stem cells with their easily accessible features and potential use for bladder reconstruction are considered ideal seed cells. ${ }^{6,7}$

Adipose Derived Stem Cells (ADSCs), which are adult stem cells, possess multiple advantages including being autologous, easy access, cellular sufficiency, multi-directional differentiation and stable gene expression. Thus, we chose ADSCs as the seed cells in the current study. Apart from seed cells, an ideal scaffold is another nuclear element for bladder reconstruction via tissue engineering technology. Bladder acellular matrix

Department of Urology, Shanghai Sixth People's Hospital, School of Medicine, Shanghai Jiaotong University, 600 Yishan Road, Shanghai 200233, China. E-mail: zhuweidong-1981@163.com grafts (BAMGs) derived from the bladder, presenting good biocompatibility and cell adhesiveness, have been widely applied in tissue engineering for bladder reconstruction. ${ }^{8}$ In our previous study, we seeded artless ADSCs onto BAMGs and successfully repaired bladder defects in a rabbit model, which confirmed the feasibility of utilizing ADSC-seeded BAMGs for partial bladder regeneration. ${ }^{9}$ However, it has been demonstrated that implantation tissues subjected to ischemia and hypoxia lead to apoptosis and ultimately affect the regeneration of smooth muscle, resulting in dysfunction. ${ }^{\mathbf{1 0}}$ Therefore, the key factor in bladder regeneration is rapidly establishing a blood supply for the transplanted scaffold.

Vascular endothelial growth factor (VEGF), belonging to the family of platelet derived growth factors, exhibits the characteristic of contributing to neovascularisation. Nevertheless, considering the short half-life period of VEGF, no valid and enduring vascularisation would rapidly arise after simply adding exogenous VEGF to transplantation environments. Fortunately, nano-carrier techniques offer a feasible solution for the sustained release and activity protection of VEGF. ${ }^{\mathbf{1 1}}$ As controlled release carriers, nanospheres have unique and significant advantages: ${ }^{\mathbf{1 2 - 1 4}}$ (1) long-term growth factor release; (2) strong controllability of growth factor release; (3) co-release or ordered release of multiple growth factors; (4) protecting protein drugs with long-term biological activity. Silk fibroin (SF) 
is a kind of nano-carrier, which has diverse biological superiorities, such as a large superficial area, good biocompatibility, and biodegradation. ${ }^{\mathbf{1 5}}$ Moreover, VEGF can be released slowly by SF-nanospheres, and further promote the regeneration of blood vessels.

In our previous study, a coaxial electro spinning technique was employed to develop VEGF-SF-BAMGs and we found that it can effectively trigger angiogenesis and promote tissue repair. ${ }^{\mathbf{1 6}}$ In another study, ${ }^{9}$ we also demonstrated that seeding ADSCs onto BAMG was more suitable for bladder reconstruction than BAMG alone to promote the regeneration of smooth muscle tissue in a rabbit model. But we had encountered difficulties with insufficient angiogenesis and graft shrinkage in our studies. ${ }^{17-19}$ To overcome these shortcomings, we attempted to change our method of material modification to add ADSCs onto our composite scaffold. In this study, we investigated whether seeding ADSCs onto a composite scaffold can achieve an enhanced blood supply and solve the problems of insufficient angiogenesis, and promote large-area bladder reconstruction in a rabbit model (Fig. 1).

\section{Materials and methods}

\subsection{Isolation and culture of adipose-derived stem cells} (ADSCs)

The adipose tissues were carefully dissected from the back and inguinal region of New Zealand White rabbits under sterile conditions and cut into small pieces, then digested in $0.1 \%$ type I collagenase (Roche USA) at $37{ }^{\circ} \mathrm{C}$ for $1 \mathrm{~h}$. The digestion products were centrifuged at $1500 \mathrm{rpm}$ for $10 \mathrm{~min}$. The isolated cells were cultured in Dulbecco's modified Eagle's medium (DMEM) containing 10\% FBS and penicillin-streptomycin. After $48 \mathrm{~h}$, the cells were washed with PBS and the medium renewed. The adipose cells isolated from each rabbit were returned in its bladder construction. All animal procedures were performed in accordance with the Guidelines for Care and Use of Laboratory Animals of Shanghai Jiaotong University and approved by the Animal Ethics Committee of Shanghai Jiaotong University Affiliated Sixth People's Hospital.

\subsection{Flow cytometry analysis and directional differentiation of ADSCs}

For cytometry analysis, fourth passage ADSCs were incubated with $10 \mu \mathrm{L}$ of individual IO test monoclonal primary antibodies coupled to either phycoerythrin (PE) or fluorescein isothiocyanate (FITC) in $50 \mu \mathrm{L}$ of PBS for $30 \mathrm{~min}$ in the dark at room temperature. Then the cells were washed twice with $1 \mathrm{~mL}$ of PBS, resuspended with PBS, diluted in $200 \mu \mathrm{L}$ of PBS and analyzed with a FACScan flow cytometer. Data were acquired using CellQuest Pro and analyzed using FlowJo software (Becton-Dickinson). Antibodies for CD44, CD29, CD90, CD31 and CD45 were obtained from BD Biosciences.

By culturing the cells under conditions that were favourable for adipogenic, chondrogenic, or osteogenic differentiation, ${ }^{\mathbf{2 0}}$ a differentiation potential was induced in the expanded mesenchymal cell at passage 3 . And adipogenesis was indicated by the accumulation of neutral lipid vacuoles that were stained with Oil Red O after 14 days of induction. Osteogenesis was indicated by an increase in calcium deposition stained by Alizarin Red after 21 days of induction. For chondrogenesis, $2 \times$ $10^{5}$ ADSCs were centrifugated into pellets. After being induced for 21 days, the pellets were sliced and stained with Alcian blue.

\subsection{Virus infection of ADSCs and cell growth assay}

A recombinant virus supernatant containing $\mathrm{MOI}=100$ was added to the ADSCs, and cultured in medium under $5 \% \mathrm{CO}_{2}$ at $37^{\circ} \mathrm{C}$. The medium was renewed after $12 \mathrm{~h}$. After being cultured for an additional $72 \mathrm{~h}$, the expression of enhanced green fluorescent protein (EGFP) was observed with a fluorescence microscope. Cell suspensions $\left(1 \times 10^{4}\right.$ per $\left.\mathrm{mL}\right)$ were seeded into 24 -well plates in DMEM medium containing $10 \%$ FBS. The number of cells was calculated by using a hematocytometer on days 3,5 and 7 .

\subsection{Construction of silk fibroin (SF) nanometer saccule}

Silkworm cocoon was boiled in aqueous solution with $0.02 \mathrm{M}$ sodium carbonate for $60 \mathrm{~min}$. The degummed silk fibers were washed with distilled water and dissolved in $9.3 \mathrm{M}$ lithium bromide solution at $60{ }^{\circ} \mathrm{C}$ for $4 \mathrm{~h}$. The solution was dialyzed

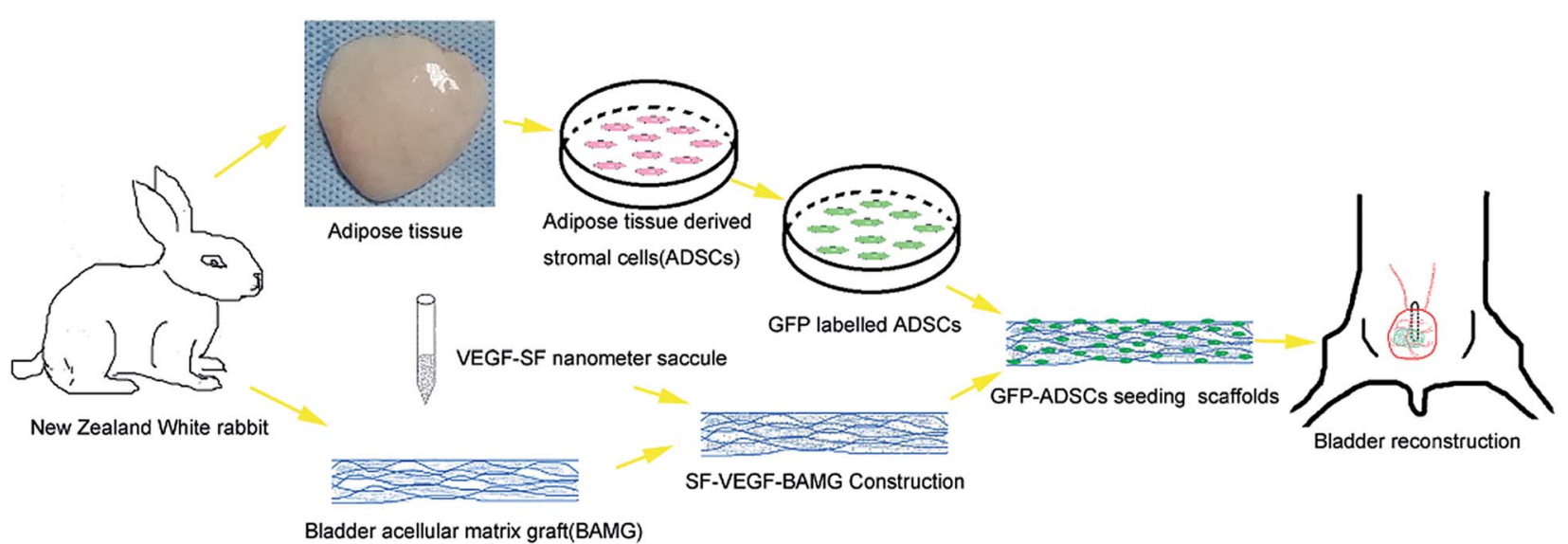

Fig. 1 Schematic diagram of tissue-engineered bladder reconstruction using a modified BAMG composite scaffold. 
against distilled water in a 12 000-14000 Da cut-off dialysis tube for $72 \mathrm{~h}$ and was further purified by centrifuging at $6000 \mathrm{rpm}$ for $15 \mathrm{~min}$. The final concentration of the aqueous SF solution was adjusted to $4 \%$ (wt/v). Then, VEGF $(10 \mu \mathrm{g})$ was dissolved in $20 \mathrm{~mL}$ of $3 \%$ (wt/v) SF solution. $8 \mathrm{~mL}$ of ethanol was added at $25{ }^{\circ} \mathrm{C}$ with constant stirring at a low rpm and stabilized with stirring at a low rpm for $5 \mathrm{~min}$. The mixed solution was frozen for $24 \mathrm{~h}$ at $-20{ }^{\circ} \mathrm{C}$ to obtain the nanoparticle solution. The nanoparticle solution was centrifuged at $40000 \times g$ for $5 \mathrm{~min}$. The SF nanoparticles were lyophilized to obtain the freeze-dried nanoparticles. A scanning electron microscope (VegaII XMU instrument Tescan, Czech-Republic) was used to observe the morphology of the SF nanometer saccule.

\subsection{Preparing the BAMGs and Masson staining}

Bladders from New Zealand White rabbits (weight: $2.5-3 \mathrm{~kg}$ ) were obtained and rinsed with PBS. The tissues were placed in $0.1 \%$ Triton X-100 (Sigma) and $0.15 \%$ (v/v) ammonium hydroxide. The solution was refreshed regularly, and after 14 days it was frozen for $24 \mathrm{~h}$ at $-80{ }^{\circ} \mathrm{C}$ and then dried for an additional $24 \mathrm{~h}$ under vacuum. Some samples were prepared to observe collagen fibers by Masson staining. Others were stored under $70 \%$ ethanol.

\subsection{Construction of VEGF-SF nanospheres-BAMG scaffolds and mechanical property study}

BAMG solution was added into 1\% (w/v) SF-VEGF solution, and ultrasonically oscillated for $5 \mathrm{~min}$. Then SF-VEGF-BAMG scaffolds were constructed through low-temperature drying under pressure. Fresh bladders, the composite scaffolds and the cellseeded scaffolds were prepared as longitudinal strips $(20 \mathrm{~mm}$ in length, $10 \mathrm{~mm}$ in width). Before testing, these strips were kept in serum-free medium at $37^{\circ} \mathrm{C}$. The mechanical properties were measured using an Instron 2710 system (INSTRON Inc. Canton, MA) at a constant speed of $10 \mathrm{~mm} \mathrm{~min}^{-1}$. With the assistance of the manufacturer's software, the ultimate loads were recorded and analyzed. The Young's modulus was calculated from the slope of the initial linear section of the stressstrain curve. Each group was tested in triplicate.

\subsection{VEGF release study and angiogenesis assays in vitro}

The release behaviours of VEGF were quantified by ELISA according to the manufacturer's instructions at each selected time point (1, 2, 3, 4, 5, 6 and 7 weeks). Briefly, VEGF-loaded samples $(n=3)$ with a weight of $100 \mathrm{mg}$ were immersed into $1 \mathrm{~mL}$ of Hank's balanced salt solutions (HBSS) and incubated in a shaking bath (100 rpm) at $37^{\circ} \mathrm{C}$. At each selected time point, a total of $400 \mu \mathrm{L}$ of supernatant was collected and replaced with an equal volume of fresh HBSS. All the samples were collected and stored at $-80{ }^{\circ} \mathrm{C}$.

To determine the functional activity of VEGF, ELISA and angiogenesis assays were performed at designated time points. Briefly, a 96-well plate was coated with $50 \mu \mathrm{L}$ of Matrigel (Sigma, MA), which was allowed to solidify at $37^{\circ} \mathrm{C}$ for $1 \mathrm{~h}$. HUVECs $(1.5$ $\times 10^{4}$ cells per well) were incubated for $20 \mathrm{~h}$, and complete tubes from randomly chosen fields were captured. Tube numbers were analysed using Image J software.

Rabbit ADSCs were cultured in the upper chamber of an HTS-Transwell-24-well microplate (Corning) and incubated with the previously collected solutions in the lower chamber for $20 \mathrm{~h}$ at $37{ }^{\circ} \mathrm{C}$ under $5 \% \mathrm{CO}_{2}$, stained with $0.1 \%$ Crystal violet, then gently wiped off the upper layer of the cell and washed with PBS 3 times. The cells were observed under a microscope in three visual fields and counted.

\subsection{ADSCs seeding onto scaffolds}

Composite scaffolds were cut into circular patches of $3 \mathrm{~cm}$ in diameter and processed with epoxyethane gas sterilization. After dunking in phosphate buffered saline (PBS) three times, the patches were soaked in $10 \mathrm{~cm}$ Petri dishes containing nonserum DMEM overnight. On the following day, the third passage ADSCs were seeded onto the scaffolds with a density of $5 \times 10^{6}$ cells per $\mathrm{cm}^{2}$, and cultured in vitro for 7 days.

\subsection{Bladder reconstruction}

Animals were randomly divided into two groups, with nine New Zealand White rabbits in each group: Group 1, the control group, were given VEGF-SF nanospheres-BAMG for bladder reconstruction; Group 2, the experimental group, were given VEGF-SF nanospheres-BAMG plus EGFP modified ADSCs for bladder reconstruction. Through a midline lower abdominal incision, about a $3 \mathrm{~cm}$-diameter full-thickness defect was dissected in the dome bladder and fully exposed under sterile conditions. 10F silica tubes with a side port were inserted anterogradely into the external urethral orifice. $3 \mathrm{~cm}$-diameter composite scaffolds which were prepared by different methods for each group were placed in the repaired sites. Their ends were continuously sutured with 5-0 absorbable thread. The corners of the BAMG were marked with a nonabsorbable suture. The fat, omentum majus and skin were closed layer by layer after hemostasis.

\subsection{Measurement of bladder capacity and morphometric analysis}

The bladder capacity before surgery and 1, 2, 3, 6 months after surgery was measured using a 7 Fr double-lumen transurethral catheter. After removing residual urine from the bladder, the bladder was filled with a pre-warmed saline solution at a constant rate. When fluid leakage was observed around the catheter, the maximal bladder capacity was defined as the volume of infusion. To determine the contracture rate of the scaffolds, the graft size at 6 months post-surgery was evaluated by measuring the distance between the non-absorbable polypropylene sutures, and then the areas were calculated and analysed with repair areas.

\subsection{Histological evaluation}

At 1, 3, 6 months after surgery, three animals were sacrificed for each time point. For HE staining, the entire bladder was removed and specimens were taken from the dome bladder to 
evaluate smooth muscle tissue regeneration. $5 \mu \mathrm{m}$ sections of formalin-fixed, paraffin wax-embedded tissues were stained with H\&E. For immunohistochemical staining, sections were deparaffinized, blocked and incubated at $4{ }^{\circ} \mathrm{C}$ overnight with anti-CD31 antibodies. After washing with PBS, sections were incubated with biotinylated secondary antibodies for $1 \mathrm{~h}$ at room temperature. Diaminobenzidine (DAB kit) was applied to as a chromogen and slides were counterstained with hematoxylin. The amount of vascular structure was evaluated by calculating the number of luminal structures, respectively, with immunohistochemical staining. All the slides were randomized using secret codes and evaluated blindly by a pathologist.

\subsection{EGFP-ADSCs tracking}

Some specimens taken from the experimental group's dome bladder were frozen to make sections at 1, 3, 6 months after surgery. After staining with 4',6-diamidino-2-phenylindole (DAPI), the sections were observed with a fluorescence microscope. The mean fluorescence intensity of EGFP was measured and calculated by Image J software according to the manufacturer's instructions.

\subsection{Statistical methods}

All of the quantitative data were expressed with means \pm SD. The difference between the groups was analyzed by one-way ANOVA. $P<0.05$ was considered statistically significant.

\section{Results}

\subsection{Identification and virus transfection of ADSCs}

After being isolated and cultured in DMEM medium containing $10 \%$ FBS for 5 days, ADSCs became fusiform and exhibited spindle shaped morphologies (Fig. 2). Flow cytometry showed that expressions of CD44 (93.4\%), CD29 (92.1\%) and CD90 (93.8\%) were higher than 92\%, while expressions of CD31 (1.5\%) and CD45 (1.8\%) were less than $2 \%$, which conformed to internationally recognized standards of ADSCs (Fig. 2). The potential of the mesenchymal cells differentiating into adipogenic, osteogenic, and chondrogenic lineages was successfully proved in Fig. 2.

To monitor the growth process of ADSCs during bladder reconstruction, a virus infection was applied to label the ADSCs with EGFP. Fig. 2 also indicates that the cells grew well and the form of the cells was normal after EGFP transfection. Cell proliferation was determined by a hematocytometer and showed no difference before and after transfection, suggesting that EGFP did not affect ADSCs proliferation.

\subsection{Construction of nano-composite scaffold}

The VEGF-SF nanometer saccules showed smooth, homogeneous and spherical-shaped images in the nano range using a scanning electron microscope (Fig. 3A and B). There was no aggregation. The diameter range of the nanometer saccules was 200-1000 nm. We selected properly sized and uniform VEGF-SF nanometer saccules to perform the following experiments. Fig. 3C shows that VEGF-SF nanometer saccules were tightly
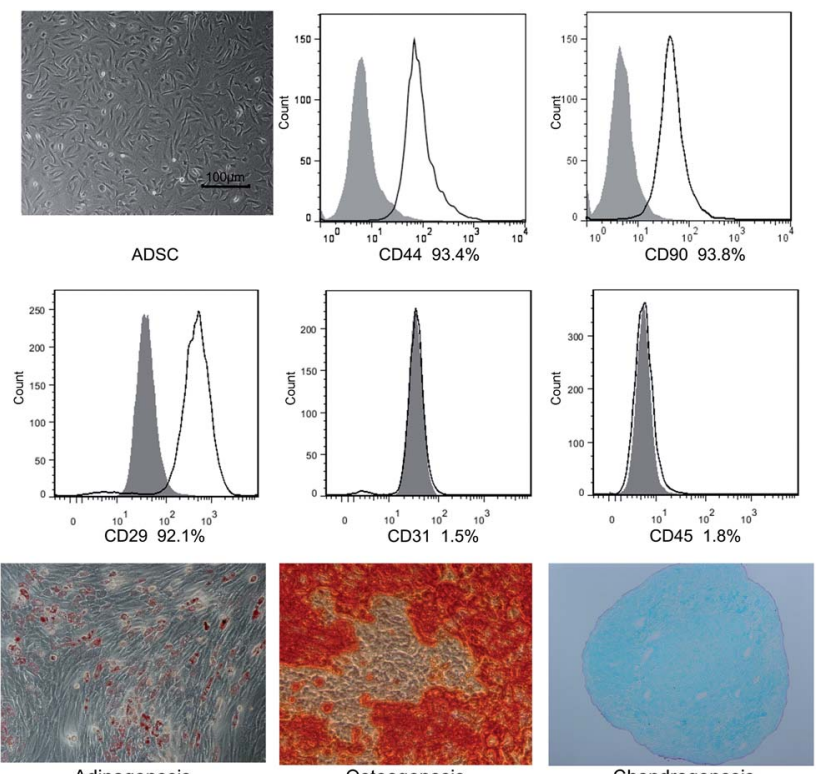

Adipogenesis

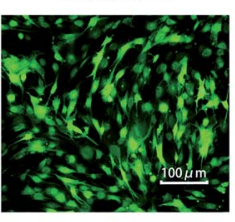

GFP

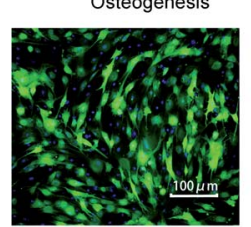

$\operatorname{Merged(DAPI)}$
Chondrogenesis

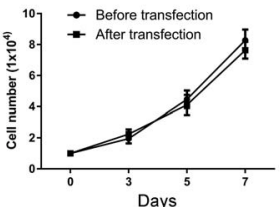

Fig. 2 Identification and virus transfection of ADSCs. ADSCs exhibited spindle shaped morphologies. Expressions of CD44, CD29 and CD90 were positive, while expressions of CD31 and CD45 were negative. The ADSCs were successfully differentiated into adipogenic $(\times 100)$, osteogenic $(\times 100)$, and chondrogenic $(\times 200)$ lineages. The ADSCs labelled with EGFP were observed with a fluorescence microscope and no difference in cell proliferation was seen.

bonded to the fibers of BAMG and were likely to support cell attachment.

Tensile stress-strain curves of fresh bladders and composited scaffolds without and with ADSCs samples are shown in Fig. 3D. The fresh bladders and composited scaffolds with ADSCs showed good response in terms of Young's modulus $(1.94 \pm 0.09$ vs. $2.37 \pm 0.24$ vs. $4.14 \pm 0.21 \mathrm{MPa})$ and stress at break $(0.21 \pm 0.03$ vs. $0.25 \pm 0.02$ vs. $0.51 \pm 0.04 \mathrm{MPa})$, which were better than the values for composited scaffolds without ADSCs $(P<0.05)$. In addition, composited scaffolds without ADSCs had higher strain at break measurements than cells with ADSCs (67.67\% vs. $75.33 \%$ ), which were closer to normal rabbit bladder cells (88.67\%). Masson staining showed no residual cell membranes or nuclear structure BAMG. The BAMG was polyporous and the bladder structure was also well preserved, which is beneficial to cell implantation (Fig. 3E).

The third generation of ADSCs were seeded onto VEGF-SF nanospheres-BAMG scaffolds and cultured for 7 days. $\mathrm{HE}$ staining and electron microscope scanning showed that the ADSCs grew well, were adsorbed in a stable manner on the nano-composite scaffold and formed multilayers on the scaffolds (Fig. 3F and $\mathrm{G}$ ). The release profile indicated that sustained release of VEGF can last for 7 weeks and reached a peak at 2 to 3 weeks, then declined relatively slowly (Fig. $3 \mathrm{H}$ ). In 

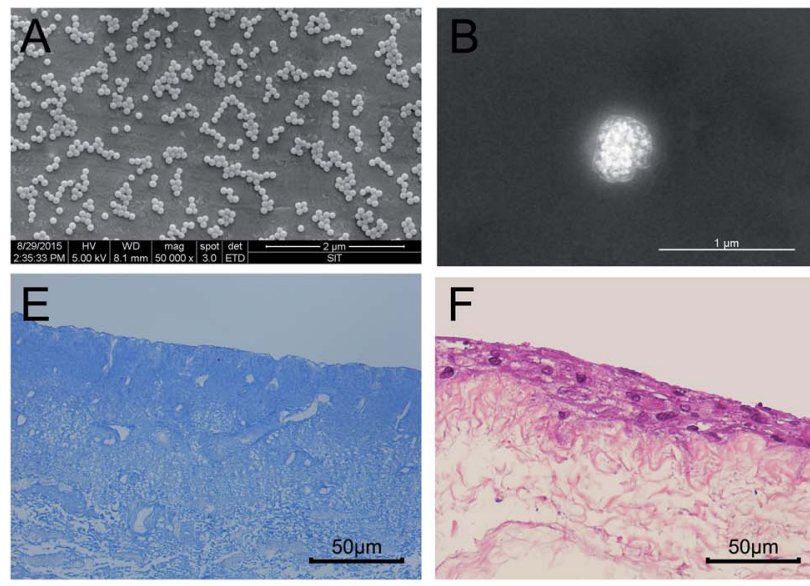
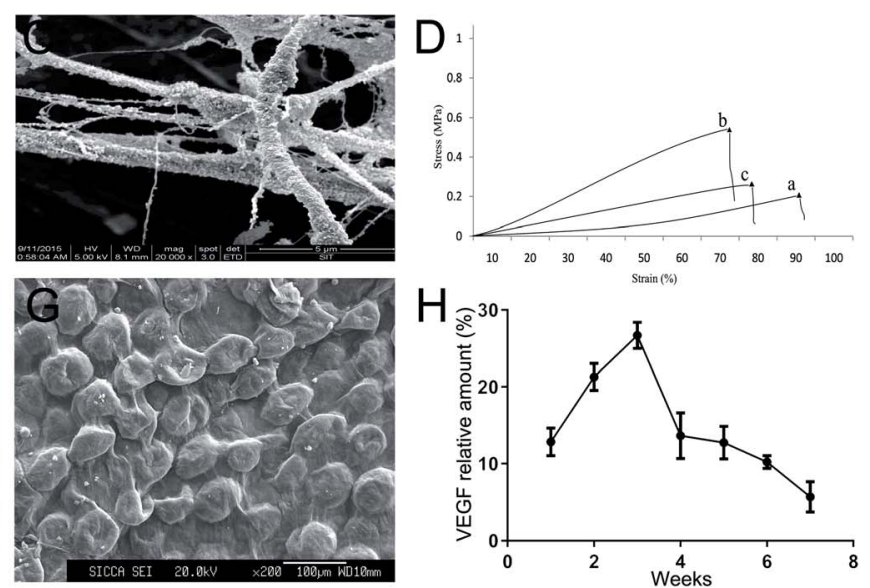

Fig. 3 Construction of nano-composite scaffold. (A and B) Scanning electron microscope (SEM) images of SF nanometer saccule; (C) SF nanometer saccule adhered to composite scaffold; (D) tensile stress-strain curves of fresh bladders (a) and composited scaffolds without (b) and with ADSCs (c); (E) Masson staining of BAMG; (F and G) ADSCs formed multilayers on the BAMG (HE, SEM); (H) release curve of VEGF from the nanometer saccules.

general, more than $60 \%$ of VEGF was released from the scaffolds in 4 weeks when detected by ELISA, suggesting a sustained release of a high concentration of VEGF.

\subsection{Angiogenesis assays}

Compared with the negative control, the VEGF released at different time points (1, 2, 3, 4, 5, 6 and 7 weeks) significantly enhanced tube numbers and networks of HUVECs and increased the migration of ADSCs (Fig. 4). The angiogenesis effect on tube formation and migration of stem cells was continuously strong from the 2 nd week to the 5 th week. It is the release behaviour of VEGF that made a difference in the tube numbers and the migration of ADSCs, and until the 7th week, there was still enough active VEGF to support angiogenesis.

\subsection{Morphometric analysis}

The operations were carried out successfully, and the effects of bladder reconstruction in the two groups after 6 months were satisfactory (Fig. 5A). No infection or diverticulum were formed. The contracture rate of the regenerated bladder in both groups was determined at 1 month and 6 months after surgery. For the area of regenerated bladder of the experimental group, the

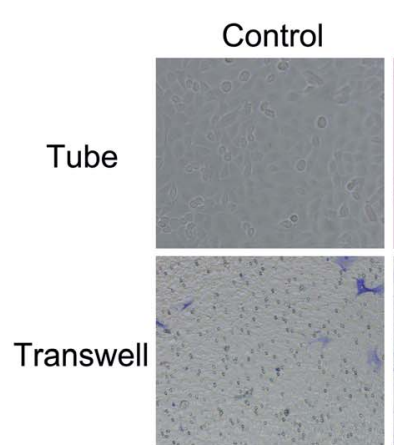

$4 \mathrm{~W}$

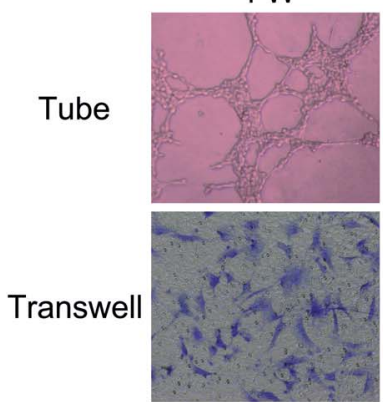

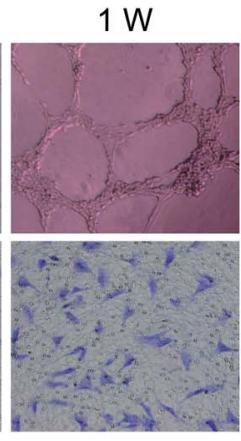

$5 \mathrm{~W}$

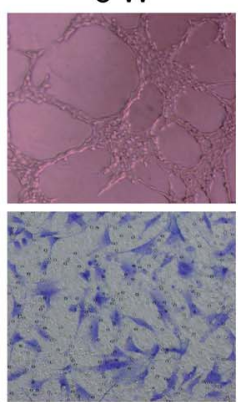

$2 \mathrm{~W}$

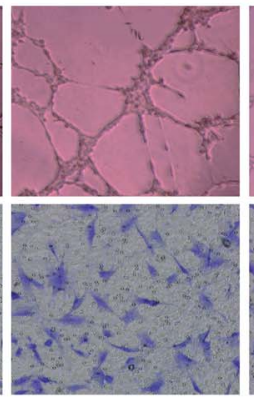

$6 \mathrm{~W}$

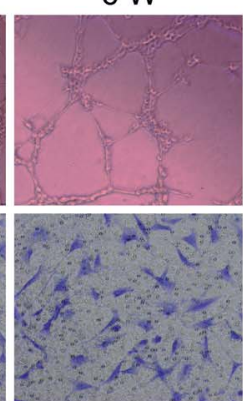

$3 \mathrm{~W}$

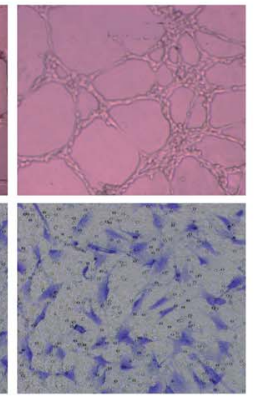

$7 \mathrm{~W}$

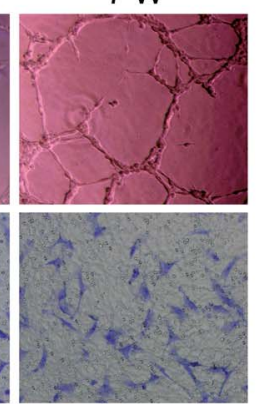

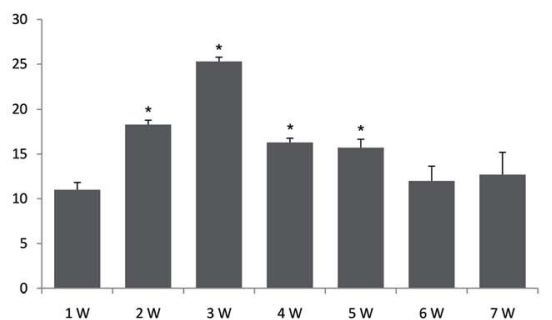

Tube formation assay

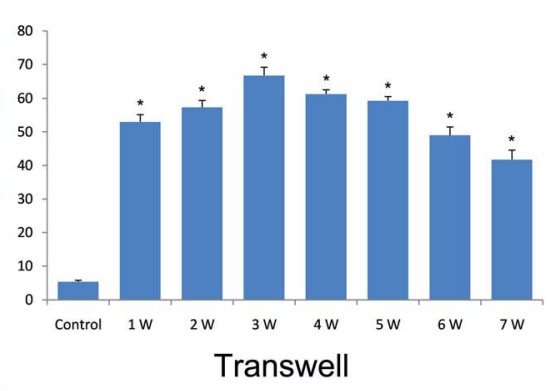

Fig. 4 Angiogenesis effect of the VEGF released in vitro $(\times 100)$. The results of tube formation and transwell assays at different time points $(1,2,3$, 4, 5, 6 and 7 weeks). 

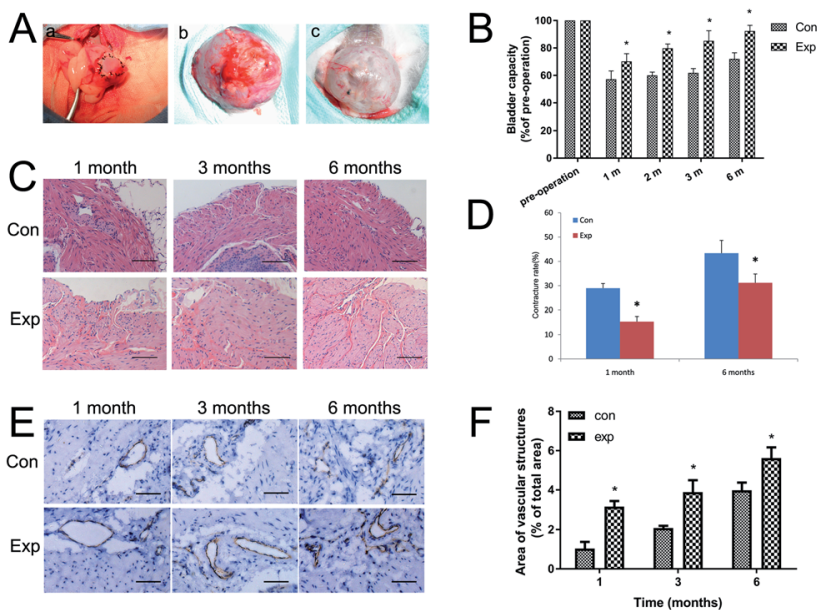

Fig. 5 Bladder capacity and histopathology evaluation. (A) (a) Surgical reconstruction of bladder defects; (b) and (c) respectively represent gross observation of the control and experimental groups 6 months after surgery. (B) Bladder capacity was evaluated preoperation and at various postoperational times in both control and experimental groups. (C) Histopathology evaluation was determined by using $\mathrm{HE}$ staining in both control and experimental groups. (D) The contracture rate in the experimental group was statistically lower than in the control group. (E) Immunohistochemical staining of CD31 in both control and experimental groups after surgery. (F) Amount of vascular structure calculated in both control and experimental groups. $* P<$ 0.05 .

contracture rate was significantly lower than that in the control group $(P<0.01)$. At 1 month postoperatively, the contracture rate was $15.32 \pm 2.15 \%$ in the experimental group and $28.96 \pm$ $1.93 \%$ in the control group. At 6 months after implantation, the contracture rate increased to $31.16 \pm 3.65 \%$ in the experimental group and $43.38 \pm 5.24 \%$ in the control group (Fig. 5D).

\subsection{Bladder capacity}

The bladder capacity in the two groups was evaluated at 1, 3, 6 months after implantation (Fig. 5B). Preoperative bladder capacity represented the $100 \%$ values, which showed no significant difference between the experimental and control groups. At 1, 3, 6 months after surgery, the bladder capacity recovery in the experimental group was better than that of the control (of pre-operation: $70.20 \% \pm 3.262 \%, 85.20 \% \pm 4.304 \%$ and $92.40 \% \pm 2.406 \%$ vs. $57.17 \% \pm 3.524 \%, 61.90 \% \pm 1.808 \%$ and $72.07 \% \pm 2.590 \%, P<0.05)$.

\subsection{Histopathology evaluation}

HE staining of harvested implants showed that, compared with the control group, the reconstructed bladder with VEGF-SF nanospheres-BAMG plus ADSCs had shown a more regular smooth muscle tissue, less inflammatory cell infiltration and scar formation (Fig. 5C), which indicated that the experimental group had a better effect on promoting the regeneration of the bladder. It was demonstrated that the amount of vascular structure gradually rose in the two groups over time. Compared with the control group, the amounts of vascular structure in the experimental group were all increased at 1, 3, 6 months post operation (Fig. 5E). The amount of vascular structure calculated at 1, 3, 6 months after surgery in the experimental group was significantly up regulated compared with the control group (Fig. 5F).

\subsection{EGFP fluorescence signal detection}

To determine the fate of the nano-composite scaffold, the new regenerated bladder wall was evaluated for EGFP-labelled cells. EGFP-labelled cells (green) were detected using a fluorescence microscope in the bladder tissue at 1, 3 and 6 months after implantation in the two groups. As shown in Fig. 6 , at 1 month, the implanted ADSCs remain dispersed in the reconstruction site. At the third month, a weaker but more uniform GFP signal was detected under a fluorescence microscope. At 6 months, the green signal was further reduced in contrast to the 1 month and 3 month values, which indicated that the expression of GFP genes in the reconstructed sites was disappearing. It seemed that the epithelial layer was thicker and the tissue structure was reconstructed in a more orderly manner.

\section{Discussion}

Tissue engineering is the most promising physiological repair technology for bladder reconstruction, ${ }^{2}$ for which, studies on seed cells and scaffold are the most important core topics. Researchers have come a long way to find a more suitable scaffold with high feasibility and cell compatibility for bladder reconstruction. To date, BAMG is the main source of material for urinary reconstruction for its inherent characteristics of the bladder and its three-dimensional space which provides an appropriate growing environment for the adhering and growing of seed cells. ${ }^{21}$ Thus, we believed that BAMG was an ideal matrix

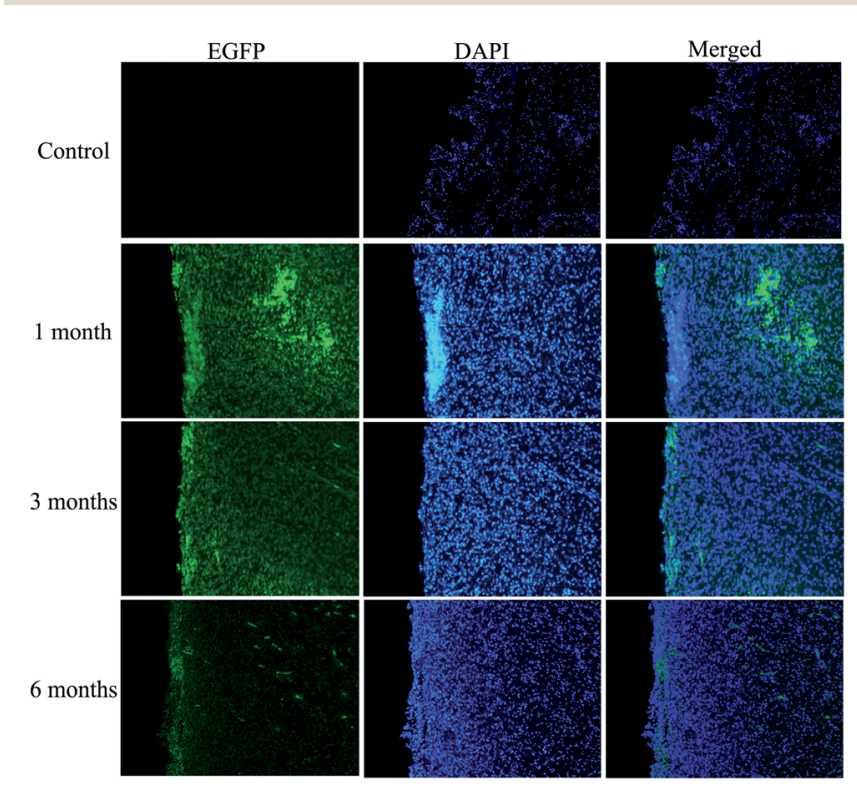

Fig. 6 EGFP fluorescence signal detection at time points $(\times 200)$. In the control group, almost no GFP signal could be found. At the first month, the survival of the ADSCs signal was high in a dispersed way. At the third and sixth months, GFP fluorescence intensity detected in the reconstruction site was reduced but more uniform. 
for bladder reconstruction and BAMG was applied in our previous study, ${ }^{9}$ the results of which indicated the promoting effect of BAMG on the regeneration of smooth muscle and nervous tissue in a rabbit model. Our subsequent studies also revealed that BAMG could repair $95 \%$ of the bladder in different bladder defects, which also demonstrated that the use of BAMG reconstruction in larger bladder defects did not induce the same quality and quantity of bladder regeneration as in the reconstruction of smaller bladder defects. Hence, in this study, we improved the property of BAMG by the method of pumping dry by a vacuum to get materials with bigger apertures and higher porosity.

Currently, how to promote regeneration of a bladder with a large area of deficiencies is a difficulty and hotspot of tissue engineering for bladder reconstruction. The biggest problem for the reconstruction of a bladder with a large area deficiency is the lack of a local blood supply. ${ }^{10}$ Early rapid vascularisation is the key element in regeneration of bladder tissue with a large area of deficiencies. VEGF, which can stimulate the release of vasoactive substances of vascular endothelial cells, could facilitate the growth and repair of tissue. ${ }^{22}$ In recent years, several researchers have focused on the combination of VEGF and scaffolds on tissue regeneration over large areas. For instance, Zhou et al. co-administrated platelet-derived growth factor-BB and VEGF with BAMG for bladder augmentation in a rabbit model, in which enhanced regeneration of smooth muscle and vascularisation were observed. ${ }^{23}$ In Zhou's study, the VEGF was prepared directly rehydrated in BAMG. Because of the instability of VEGF, it is hard to maintain an efficient angiogenesis promoting effect, and thus an appropriate vector is necessary. Accumulated studies demonstrated the feasibility of nanospheres as the vector for the growth factor. ${ }^{24}$ Jiang et al. ${ }^{19}$ constructed VEGF-loaded nanoparticle-modified BAMG in tissueengineered bladders in rabbits, which resulted in enhanced angiogenesis and inhibition of graft shrinkage. In their study, VEGF was loaded on PLGA nanoparticles. Because of the poor bio-compatibility of artificial nanoparticles, which could affect the feasibility of a composite scaffold for tissue regeneration, in our study, we applied a natural polymer-silk fibro (SF) as the vector for VEGF.

SF has good bio-security and it has been applied in tissue engineering in a large number of studies. It is a natural polymer with superior mechanical properties, high permeability to oxygen and water, low immunogenicity and great cell adhesion and growth features. ${ }^{25}$ Chiarini et al. ${ }^{26}$ revealed that SF modified PU/PCU could enhance cell binding ability and proliferation ability in in vitro cell research. In this study, we successfully constructed SF nanospheres by repeatedly attempting to find the appropriate $\mathrm{pH}$, temperature and $\mathrm{SF}$ concentration. SF nanospheres with diameters of 200-1000 nm were prepared for future experiments. By applying an ELISA assay, the continued sustained release of VEGF from SF nanospheres was observed, which was consistent with our previous study, in which VEGF was applied to combine with PLGA nanosphere modified BAMG scaffolds in rabbit anterior urethral reconstruction. ${ }^{9}$ When we constructed VEGF, which has a short half-life, with SF nanospheres, the sustained slow release of this factor achieved satisfying angiogenesis results in vitro. The consistent results of angiogenesis and migration experiments showed SF nanosphere modified BAMG scaffolds sustained the release of active VEGF for at least 7 weeks. Especially in the first month after implantation, the relative high VEGF concentration might greatly increase the chances of the full vascularisation of the grafts. In addition, both the composite scaffolds and the cellseeded scaffolds exhibited excellent mechanical properties as fresh bladders, which is vital to the recovery of bladder function.

In the present study, after ADSCs had been isolated, expanded and then identified, they were seeded onto a VEGF-SF nanosphere-BAMG composite scaffold to reconstruct bladders with large area deficiency in rabbits. To follow up the destination of ADSCs in our composite scaffolds, virus infection was adopted to label the ADSCs with EGFP. After the scaffolds were implanted into the animals, the reconstructed bladder with VEGF-SF nanosphere-BAMG plus ADSCs had a higher bladder capacity, more regular smooth muscle tissue and improved shrinkage, and more blood vessels, indicating that the method has a good effect on promoting regeneration of the bladder. Moreover, our results did not support the viewpoint that ADSCs could differentiate into epithelial or vascular endothelial cells, because cells in both the epithelial layer and vascular areas containing GFP fluorescence signals had not been found. Instead, ADSCs may possibly provide additional VEGF and others cytokines to enhance angiogenesis in the bladder regeneration process. After surgery, the bladder capacity was slowly but significantly improved over time in Group 2 compared with Group 1. Considering the slow-release behaviour of the scaffolds, it was measured monthly in the first three months. However, the capacity of the reconstructed bladder in Group 2 recovered to nearly $90 \%$ of the normal volume compared with that of $70 \%$ in Group 1 . HE and immunohistochemical staining were consistent with the results from bladder capacity. The above revealed that the nano-composite scaffold can promote regeneration of bladder tissue by means of continuous slow release of VEGF based on SF nanotechnology and combined with ADSCs, which achieved a good repair effect.

In summary, we used BAMG modified by SF nanospheres which can sustainably-release VEGF as the scaffold material, and chose ADSCs as the seed cells in this study, to perform bladder reconstruction in rabbit bladder defect models. The results in the present study indicated that the tissue engineered bladder constructed by ADSCs and BAMG modified by VEGF-SF nanospheres possessed high bio-compatibility and a good degradation rate, and can be used as an ideal biomaterial to repair the bladder.

\section{Conclusions}

The tissue engineered bladder constructed with BAMG modified by VEGF-SF nanospheres possessed high bio-compatibility and an enhanced angiogenesis effect, which can be used as an ideal biological material to repair bladder defects after being composited with ADSCs, suggesting that BAMG modified by 
VEGF-SF nanospheres represents a promising approach for clinical applications in bladder regeneration.

\section{Conflicts of interest}

There are no conflicts to declare.

\section{Acknowledgements}

This work was supported by the National Natural Science Foundation of China (Grant No. 81200497). We thank Xiangguo $\mathrm{Lv}$, Chongyang Wang and Juehong Li for their kind assistance in this study.

\section{References}

1 O. M. El-Taji, A. Q. Khattak and S. A. Hussain, Oncol. Lett., 2015, 10, 3-10.

2 S. Y. Chung, Lancet, 2006, 367, 1215-1216.

3 A. Atala, S. B. Bauer, S. Soker, J. J. Yoo and A. B. Retik, Lancet, 2006, 367, 1241-1246.

4 Y. G. Chung, K. Algarrahi, D. Franck, D. D. Tu, R. M. Adam, D. L. Kaplan, C. R. Estrada Jr and J. R. Mauney, Biomaterials, 2014, 35, 7452-7459.

5 O. Lam Van Ba, S. Aharony, O. Loutochin and J. Corcos, Adv. Drug Delivery Rev., 2015, 82-83, 31-37.

6 J. N. Lee, S. Y. Chun, H. J. Lee, Y. J. Jang, S. H. Choi, D. H. Kim, S. H. Oh, P. H. Song, J. H. Lee, J. K. Kim and T. G. Kwon, J. Korean Med. Sci., 2015, 30, 1754-1763.

7 N. A. Mousa, H. A. Abou-Taleb and H. Orabi, World J Stem Cells, 2015, 7, 815-822.

8 M. Pokrywczynska, I. Gubanska, G. Drewa and T. Drewa, BioMed Res. Int., 2015, 2015, 613439.

9 W. D. Zhu, Y. M. Xu, C. Feng, Q. Fu, L. J. Song and L. Cui, World J. Urol., 2010, 28, 493-498.

10 R. K. Jain, P. Au, J. Tam, D. G. Duda and D. Fukumura, Nat. Biotechnol., 2005, 23, 821-823.
11 H. Geng, H. Song, J. Qi and D. Cui, Nanoscale Res. Lett., 2011, 6, 312.

12 O. Jeon, S. W. Kang, H. W. Lim, J. Hyung Chung and B. S. Kim, Biomaterials, 2006, 27, 1598-1607.

13 L. Chen, L. Liu, C. Li, Y. Tan and G. Zhang, J. Nanosci. Nanotechnol., 2011, 11, 3107-3114.

14 L. Tan, R. Huang, X. Li, S. Liu and Y. M. Shen, Acta Biomater., 2017, 57, 498-510.

15 F. Mottaghitalab, M. Farokhi, M. A. Shokrgozar, F. Atyabi and H. Hosseinkhani, J. Controlled Release, 2015, 206, 161176.

16 W. Zhu, C. Feng, X. Zhang, Q. Fu, L. Song and R. Chen, J. Biomater. Tissue Eng., 2016, 6, 493-499.

17 P. A. Merguerian, P. P. Reddy, D. J. Barrieras, G. J. Wilson, K. Woodhouse, D. J. Bagli, G. A. McLorie and A. E. Khoury, BJU Int., 2000, 85, 894-898.

18 D. Rickert, M. A. Moses, A. Lendlein, S. Kelch and R. P. Franke, Clin. Hemorheol. Microcirc., 2003, 28, 175-181.

19 X. Jiang, Q. Xiong, G. Xu, H. Lin, X. Fang, D. Cui, M. Xu, F. Chen and H. Geng, Ann. Biomed. Eng., 2015, 43, 25772586.

20 M. F. Pittenger, A. M. Mackay, S. C. Beck, R. K. Jaiswal, R. Douglas, J. D. Mosca, M. A. Moorman, D. W. Simonetti, S. Craig and D. R. Marshak, Science, 1999, 284, 143-147.

21 Z. Zhe, D. Jun, Z. Yang, X. Mingxi, Z. Ke, Z. Ming, W. Zhong and L. Mujun, Stem Cells Dev., 2016, 25, 405-414.

22 R. Chung, B. K. Foster and C. J. Xian, J. Endocrinol., 2014, 221, 63-75.

23 L. Zhou, B. Yang, C. Sun, X. Qiu, Z. Sun, Y. Chen, Y. Zhang and Y. Dai, Tissue Eng., Part A, 2013, 19, 264-276.

24 S. Zhang and H. Uludag, Pharm. Res., 2009, 26, 1561-1580.

25 M. Farokhi, F. Mottaghitalab, M. A. Shokrgozar, J. Ai, J. Hadjati and M. Azami, Mater. Sci. Eng., C, 2014, 35, 401410.

26 A. Chiarini, P. Petrini, S. Bozzini, I. Dal Pra and U. Armato, Biomaterials, 2003, 24, 789-799. 